Archives

$4 \mid 1989$

Varia

\title{
Les échanges de technologie entre la France et le monde anglo-américain à l'ère de l'industrialisation : le cas des moteurs hydrauliques
}

\section{Serge Benoit}

\section{(2) OpenEdition}

12 Journals

\section{Édition électronique}

URL : http://journals.openedition.org/ccrh/2898

DOI : $10.4000 /$ ccrh.2898

ISSN : $1760-7906$

Éditeur

Centre de recherches historiques - EHESS

\section{Édition imprimée}

Date de publication : 15 octobre 1989

ISSN : 0990-9141

Référence électronique

Serge Benoit, « Les échanges de technologie entre la France et le monde anglo-américain à l'ère de I'industrialisation : le cas des moteurs hydrauliques », Les Cahiers du Centre de Recherches Historiques [En ligne], 4 | 1989, mis en ligne le 22 avril 2009, consulté le 10 décembre 2020. URL : http:// journals.openedition.org/ccrh/2898; DOI : https://doi.org/10.4000/ccrh.2898

Ce document a été généré automatiquement le 10 décembre 2020.

Article L.111-1 du Code de la propriété intellectuelle. 


\title{
Les échanges de technologie entre la France et le monde anglo-américain à l'ère de l'industrialisation: le cas des moteurs hydrauliques
}

\author{
Serge Benoit
}

\section{Le contexte d'ensemble des échanges : le rôle de l'énergie hydraulique dans la croissance au XIX ${ }^{\mathrm{e}}$ siècle}

\section{La force hydraulique, facteur majeur en de nombreux pays de la première industrialisation}

1 En tant qu'ensemble de transformations techniques, économiques et sociales, l'industrialisation est généralement présentée comme un processus ininterrompu s'étendant depuis certains pays dits initiateurs vers d'autres qualifiés de suiveurs. Ce type de schéma, impliquant des rapports de hiérarchie et de domination entre différentes formations sociales, associés à des transferts de caractère unilatéral des uns vers les autres et des décalages dans le temps quant au passage obligé par les étapes d'un même modèle de croissance, avait été plus particulièrement consacré, sur le plan de l'histoire des techniques, par le colloque international organisé en 1970 par le C.N.R.S. à Pont-àMousson $^{1}$. Celui-ci s'était tenu au terme d'une décennie qui avait vu s'affirmer, parmi les historiens et les économistes du développement, la problématique des transferts de technologie entre pays inégalement avancés ou à systèmes sociaux différents.

Dans cette optique, la première industrialisation, de la fin du XVIII ${ }^{e}$ siècle à la fin du XIX ${ }^{e}$, apparaissait caractérisée par l'existence d'un unique foyer initial - la Grande-Bretagne -, celle-ci ayant non seulement joué le rôle initial d'impulsion, mais ayant en outre apporté la quasi-totalité des composantes de base du nouveau système technique, si nous 
reprenons ici provisoirement, par commodité, la terminologie et les concepts promus en France par Bertrand Gille. On sait la position centrale attribuée par l'historiographie classique de la révolution industrielle, dans cette structure technologique du siècle dernier, à l'apparition de la machine à vapeur, en raison à la fois de ses liens, en amont, avec l'exploitation à grande échelle du combustible fossile le plus répandu, la houille, et, en aval, avec la mécanisation de la production et des transports. Suivant cette vision, l'extension de l'industrialisation s'identifiait très largement avec la diffusion de ce nouveau type de moteur primaire considéré comme l'instrument technique majeur de la croissance économique.

3 Cette présentation, qui avait été celle des contemporains, et avait ensuite massivement prévalu dans l'interprétation historique, se trouve de plus en plus remise en cause depuis une trentaine d'années par le courant international de recherches ayant mis en lumière le rôle prolongé, voire prédominant, dans de nombreuses expériences nationales ou régionales, de la force hydraulique comme base énergétique de la mécanisation industrielle. Bien avant d'avoir trouvé son emploi final, devenu presque exclusif aujourd'hui pour la production électrique, la puissance des rivières avait été appliquée à grande échelle, sous son ancestrale forme mécanique directe, pour fournir leur force motrice à nombre d'activités manufacturières spécifiques de la première industrialisation, après avoir permis l'élaboration, depuis la fin du Moyen Age jusqu'à à l'ère dite proto-industrielle, de premières formes, sans doute partielles mais non moins décisives, de mécanisation de la production.

C'est à propos des Etats-Unis que la démonstration de ce processus a été apportée avec le plus d'ampleur, à la faveur d'un ensemble de travaux d'où émergent tout particulièrement ceux du regretté Louis $C$. Hunter, auteur d'une magistrale synthèse présentée en 1979 sous le titre, encore un peu provocateur à l'époque, de Waterpower in the Century of Steam ${ }^{2}$. Non seulement l'auteur y mettait en évidence la place prépondérante tenue jusqu'à la guerre civile par l'énergie des cours d'eau dans les principales branches d'activité, à commencer par le secteur, moteur par excellence, de l'industrie cotonnière implantée en Nouvelle-Angleterre, mais en outre il faisait ressortir l'échelle, sans équivalent dans le reste du monde contemporain, à laquelle cette source de force motrice avait été mise en oeuvre, et qui aboutissait, dans les conditions du Nouveau Monde, à des effets égaux voire supérieurs à ceux de la vapeur quant à la taille des unités de production et la concentration de la main-d'oeuvre et du peuplement.

Des conclusions similaires tendent à se faire jour, au moins pour le premier de ces deux derniers aspects, en ce qui concerne la France, à la convergence d'études sectorielles d'histoire industrielle ( $\mathrm{Cl}$. Fohlen, D. Woronoff, S. Chassagne), de travaux généraux d'histoire des techniques (M. Daumas, B. Gille), et de l'essor de l'archéologie industrielle que, depuis une dizaine d'années, le Centre de Recherches Historiques a contribué, de manière décisive, sous l'impulsion de L. Bergeron, à définir et promouvoir dans sa conception française. Si, par suite de conditions notamment hydrogéographiques, bien différentes de celles prévalant Outre-Atlantique, le recours à l'énergie hydraulique n'a pu s'accompagner ici des mêmes effets polarisants, il n'en demeure pas moins qu'à l'instar des Etats-Unis, il fut prédominant jusqu'aux années 1860, à l'échelle nationale, et, dans certaines régions ou dans des secteurs déterminés, pendant encore deux, voire trois décennies.

6 Le pays de James Watt, lui-même, n'a pas échappé à cette réévaluation du rôle historique de la force hydraulique. Rectifiant l'image trop ancrée d'un triomphe de la vapeur Outre- 
Manche aussi précoce que général, reprise en droite ligne des grands classiques depuis P. Mantoux jusqu'à, plus près de nous, D.S. Landes en passant par T.S. Ashton, les travaux, d'inspiration économétrique, d'un G.N. von Tunzelmann, comme ceux, de caractère sectoriel et régional, d'un $\mathrm{S}$. Chapman, ont mis en lumière la place nullement négligeable conservée, en-dehors du Lancashire, jusqu'aux années 1820 et 1830, par la force motrice des cours d'eau. Dans le cas de l'Ecosse, la récente synthèse de J. Shaw ${ }^{3}$ a montré que plus d'un secteur industriel avait continué de faire majoritairement appel à celle-ci jusqu'aux années 1850 , en dépit des importantes ressources charbonnières de la région, dans le contexte en longue durée d'un mouvement d'équipement des rivières, aussi intense qu'ininterrompu, depuis la fin du xviI ${ }^{e}$ siècle.

7 Dans les trois cas nationaux qui viennent d'être cités, la demande industrielle en force hydraulique a suscité, à des moments différents, un important effort d'innovation pour perfectionner les moteurs captant cette énergie naturelle. On peut d'autant plus se demander, dans ces conditions, dans quelle mesure ces trois pays ont pu s'influencer mutuellement dans ce domaine, que chacun d'entre eux y fut, à tour de rôle reconnu comme leader: la Grande-Bretagne des années 1770 aux années 1820, puis la France jusqu'aux années 1850-1860, enfin les Etats-Unis jusqu'au tournant du siècle dernier. S'il ne faut pas perdre de vue que ce mouvement créatif a associé d'autres Etats et donné lieu à bien d'autres transferts vers un grand nombre d'autres régions européennes, c'est dans la mesure où ces trois pays ont joué successivement un rôle de leader - ainsi que pour des raisons de commodité d'accès à la littérature historique -, qu'ils ont paru présenter ici un intérêt particulier comme exemple de relations triangulaires au cours de la période envisagée.

8 Pour saisir pleinement la portée de ces échanges, il est indispensable de rappeler, au préalable, le point de départ de l'évolution dans le cadre de laquelle ils se sont inscrits, c'est-à-dire l'état de la technologie des moteurs hydrauliques en Europe et en Amérique du Nord au milieu du XvIII ${ }^{e}$ siècle.

\section{L'état d'ensemble de la technologie hydraulique aux commencements de la révolution industrielle.}

Jusqu'à cette époque, en effet, on avait disposé de deux classes de récepteurs hydrauliques, employés les uns et les autres sur les deux rives de l'Atlantique, selon toutefois une distribution géographique très inégale: les roues dites verticales tournant autour d'un axe horizontal, et celles appelées horizontales effectuant leur rotation autour d'un axe vertical. Dans le contexte de la proto-industrialisation, les roues verticales avaient davantage retenu l'attention des techniciens et des utilisateurs, dans la mesure où elles seules pouvaient développer des puissances unitaires de quelques chevaux à quelques dizaines de chevaux (de un à une vingtaine de $\mathrm{KW}$ en termes contemporains), celles-là mêmes que requéraient les formes déjà partiellement mécanisées de la production manufacturière (papeterie, sidérurgie, foulonnage des étoffes pour ne citer que les plus importantes). Les roues horizontales n'atteignaient, en revanche, que quelques chevaux au plus, et ne pouvaient guère, dans ces conditions, être employées qu'à la mouture des grains, ou au sciage du bois, malgré certaines applications aux stades d'une fabrication industrielle déjà aussi complexe que la soierie, qui, comme le moulinage, n'exigeaient que de faibles besoins énergétiques. Qu'elles fussent horizontales ou verticales, ces roues, toutes construites pour l'essentiel en bois, ne pouvaient 
développer que des puissances limitées et leur durée de vie n'excédait pas en moyenne 10 à 15 ans.

Les roues verticales se subdivisaient elles-mêmes en deux grandes familles, en fonction de la position d'arrivée du courant d'eau motrice: celles mues par dessous et celles actionnées par dessus, les undershot et les overshot waterwheels dans la terminologie angloaméricaine. L'adoption, par l'utilisateur, des unes ou des autres était avant tout conditionnée par les conditions topographiques et, à certains égards, hydrologiques. Les roues en dessous convenaient aux plus basses chutes, inférieures en pratique à deux mètres de hauteur, sur des rivières offrant un débit relativement abondant; celles en dessus s'imposaient pour des chutes de plus de trois mètres, sur des cours d'eau peu importants voire de simples ruisseaux. Ce fut précisément, au milieu du xvIII siècle, qu'une clarification discriminante fut enfin apportée pour l'appréciation des avantages et des inconvénients respectifs des unes et des autres, sur lesquels les techniciens étaient restés très partagés au cours des décennies antérieures. Presque simultanément, entre 1751 et 1754, une série d'expérimentations réalisées en Grande-Bretagne par John Smeaton et en France par Antoine Deparcieux établirent rigoureusement la supériorité, sur le plan du rendement, des roues en dessus mues par le poids de l'eau sur celles en dessous entraînées par le choc du courant ${ }^{4}$. L'effet utile prouvé des premières atteignait 50 à $60 \%$ de la puissance brute de la chute, contre seulement 25 à $30 \%$ dans le cas des secondes. Parallèlement, les recherches de caractère physico-mathématique poursuivies tout au long du siècle à travers l'Europe en matière de mécanique rationnelle des fluides, depuis Edme Mariotte jusqu'à Lagrange, en passant par Daniel Bernoulli, Antoine Parent, Leonhard Euler et Jean-Charles Borda, permirent de mieux comprendre analytiquement le fonctionnement des moteurs hydrauliques et de poser, notamment à la faveur des travaux du dernier, les principes fondamentaux de leur perfectionnement.

11 Les roues horizontales ne furent cependant pas complètement délaissées. Elles comprenaient elles-mêmes deux grandes catégories, qui se distinguaient selon le mode d'action de l'eau. Aux roues à cuillers, mues par l'impulsion du courant, s'opposaient les roues à cuves, actionnées par la réaction du poids de l'eau. Le traité de Bernard Forest de Belidor, L'Architecture hydraulique, célèbre malgré les nombreuses erreurs qu'il comporte, et publié pour la première fois en 1737, en avait souligné l'avantage majeur, résidant dans l'absence de tout organe de transmission, pour l'entraînement des moulins à céréales, dans la mesure où la meule tournante pouvait être montée directement sur l'arbre de rotation de la roue. Il avait non moins insisté sur la capacité des roues à cuve à fonctionner noyées en cas de crues, ce qui levait l'un des principaux handicaps reprochés aux moteurs hydrauliques, c'est-à-dire leurs défaillances liées aux variations saisonnières du niveau des eaux. Au début des années 1750, par ailleurs, l'illustre mathématicien suisse Leonhard Euler exposa, dans un mémoire à l'Académie de Berlin, la théorie d'une nouvelle roue horizontale susceptible d'atteindre le maximum de rendement.

12 En dépit de ces nombreux développements scientifiques, ce ne fut pas sur le continent européen, mais en Grande-Bretagne qu'intervinrent, durant la seconde moitié du XVIII ${ }^{\mathrm{e}}$ siècle, une première série de progrès majeurs dans la technologie des moteurs hydrauliques. Ils résultèrent non pas des avancées de la théorie, mais de la pratique expérimentale parfaitement maîtrisée de Smeaton et de ses émules. Ils furent la réponse aux besoins énergétiques suscités par un essor de la production manufacturière sans équivalent par son intensité, et par celui des nouvelles activités fondatrices de la première révolution industrielle, comprenant au premier chef la filature de coton 
mécanisée et la sidérurgie à la houille, celles-là mêmes qui, au même moment en GrandeBretagne aussi, constituèrent l'incitation décisive dans la mise au point du premier moteur thermique de l'histoire aux applications universelles: la machine à vapeur de Watt.

\section{Les grandes étapes des échanges entre Grande- Bretagne, France et États-Unis dans le domaine des moteurs hydrauliques, de la fin du XVIII à la fin du XIX siècle}

\section{Le transfert initial à partir de la Grande-Bretagne : les roues de côté à augets (v. 1770 - v. 1830)}

13 Pressés par une demande exceptionnellement intense, telle, qu'en bien des contrées, l'équipement des rivières en sites hydrauliques avait atteint Outre-Manche un véritable point de saturation, les mécaniciens britanniques parvinrent, en quelques décennies, à mettre au point, par touches successives, des solutions qui s'avérèrent capables de répondre, pour plus d'un demi-siècle, aux exigences des industriels disposant de la force hydraulique.

14 Les principaux développements technologiques de la seconde moitié du XVIII ${ }^{\mathrm{e}}$ siècle, en Grande-Bretagne, dérivèrent en ligne directe des travaux de Smeaton. Leurs auteurs s'employèrent à perfectionner la roue en dessus, et surtout à en rendre le principe applicable dans le cas de chutes, les plus répandues géographiquement, plus basses que celles sous lesquelles elles fonctionnaient ordinairement. Ils aboutirent ainsi à la formule de la roue de côté à augets emboîtée dans un coursier courbe, qui, en faisant agir l'eau partiellement par son poids, apportait une substantielle amélioration de rendement par rapport aux roues en dessous traditionnelles, jusqu'à 40 et $50 \%$, pour une gamme de chutes de 1 à 5-6 m. Elle permettait, en même temps, de se dégager des contraintes mécaniques inhérentes à la roue en dessus, qui imposaient ainsi un diamètre pratiquement équivalent à la hauteur de chute, avec tous les inconvénients susceptibles d'en résulter quant au positionnement de l'axe et à la vitesse de rotation.

$\mathrm{Au}$ début du XIX ${ }^{e}$ siècle, la roue de côté (ainsi appelée lorsque l'eau était admise en un point situé entre la partie inférieure de la roue et l'arbre) et sa version dite de poitrine (lorsque l'eau pénétrait dans la roue en un point situé entre son sommet et le niveau de l'axe), reçurent une nouvelle série de perfectionnements appréciables, qui en relevèrent encore sensiblement l'effet utile. L'adoption de la vanne inclinée à déversement fournit l'indispensable complément au profil courbe du coursier pour améliorer l'admission de l'eau dans la roue; l'on s'employa également à adapter aux roues hydrauliques le régulateur à boules mis au point par Watt pour égaliser le mouvement des machines à vapeur. Plus encore, ce fut dans les mêmes années que s'affirma, Outre-Manche, la tendance à construire ces roues perfectionnées en métal, partiellement ou en totalité, à la faveur des nouveaux procédés de la métallurgie apparus en Grande-Bretagne - la fonderie de seconde fusion et le laminage combinés avec l'emploi du coke et de la houille -, qui permettaient de travailler avec beaucoup plus de souplesse et à bien moindre coût, la fonte et le fer, tout en obtenant des produits sans cesse diversifiés et plus élaborés. L'innovation technologique représentée par la construction métallique n'était pas la 
moins importante de toutes : elle permettait, en effet, à la fois d'accroître de plusieurs fois la durée de vie des roues (certaines restèrent ainsi en service pendant 140 ans !) et, tout autant, d'en élever la puissance unitaire à plusieurs dizaines, et même plusieurs centaines de chevaux.

Portés à leur forme canonique par des mécaniciens tels que John Rennie, Thomas Hewes et William Fairbairn, ces types de roues perfectionnées mis au point en Grande-Bretagne y devinrent l'instrument de base de celles des manufactures qui, malgré l'ascension rapide et continue de la vapeur Outre-Manche, continuaient de faire appel à l'hydraulique. Dans le secteur textile notamment, elles auraient, selon certains témoignages contemporains, supplanté complètement les types de roues antérieurs, endessous mais même aussi en-dessus ${ }^{5}$.

En France, ces roues à l'anglaise firent leur apparition durant les premières années de l'ère napoléonienne, autour de 1805 , mais ne se diffusèrent véritablement sous leurs caractéristiques majeures qu'après 1815 . Ce fut à l'époque de la Restauration qu'elles furent le plus proche du modèle britannique. Sous la Monarchie de Juillet, la roue de côté se vulgarisa massivement, mais sous des formes plus rustiques, qui maintenaient une plus grande part au bois dans sa structure; l'emploi d'aubes droites fut, de même, assez souvent préféré à celui d'augets, mais la vanne plongeante tendit à se généraliser dans bien des contrées. Ici aussi, la roue de côté fut, jusqu'aux années 1840 , le principal outil de la modernisation des installations hydrauliques, quant au nombre de sites sur lesquels elle fut adoptée.

Outre-Atlantique, vers 1805 , la configuration de côté ou de poitrine était apparemment déjà plus courante qu'en France, même si la facture des roues elle-même restait largement de type traditionnel, c'est-à-dire réalisée massivement en bois, conformément aux tendances durables de la technologie Nord-américaine incitée à recourir à un matériau naturel aussi bon marché qu'abondant. Là encore, cependant, ce fut après 1815 que se multiplièrent les modèles les plus typiquement influencés par les conceptions britanniques, notamment pour les roues destinées à l'industrie textile dans la construction desquelles le métal entra cette fois de manière prépondérante sinon exclusive. Compte tenu du gigantisme déjà atteint par un certain nombre de ces cottonmills, à la mesure des rivières ou des fleuves de cette façade océanique du NouveauMonde, elles ne tardèrent pas à dépasser largement, pour la puissance unitaire moyenne, celles de Grande-Bretagne, et à approcher les 300 chevaux pour les plus importantes d'entre elles. Jusqu'à l'approche du milieu du siècle, les roues de poitrine (high breast) régnèrent sans partage dans les grands centres textiles de l'arrière-pays bostonien et du Centre-Est atlantique. Leur domination fut alors remise en question par une nouvelle venue d'origine principalement française : la turbine, en l'espèce sous ses deux premières formes centrifuge et axiale.

\section{Le rayonnement international des turbines d'origine française, 1830-1860}

Ce fut après 1815 , au milieu des années 1820 , que la capitalisation des acquis scientifiques hérités du XVIII siècle, plus avancée en France qu'ailleurs en matière d'hydraulique, jointe à l'introduction des innovations métallurgiques britanniques sur le plan technologique, aboutit de ce côté-ci de la Manche à une percée technique majeure, avec la mise au point de la turbine. Celle-ci marquait non seulement l'avènement d'une nouvelle 
catégorie de récepteurs hydrauliques, mais d'une classe générique de moteurs primaires appelés aux immenses développements que l'on sait au $\mathrm{xx}^{\mathrm{e}}$ siècle : les turbomachines.

Apparue en 1827 sous la forme d'un simple démonstrateur, et en version opérationnelle à partir de 1830, la turbine centrifuge réalisée par Benoît Fourneyron d'après un schéma dû en fait à Claude Burdin, représentait la première concrétisation de cette roue horizontale à hautes performances que les techniciens avaient appelée de leurs voeux depuis le milieu $\mathrm{du}$ siècle précédent. Elle différait sensiblement dans son principe du schéma originellement imaginé par Leonhard Euler, pour se rapprocher davantage de celui mis en oeuvre dans les roues à réaction, essayées depuis la fin du XVIII ${ }^{\mathrm{e}}$ siècle en France et surtout en Grande-Bretagne, qui appliquaient les effets de couple du tourniquet hydraulique. L'eau traversait de l'intérieur vers la périphérie deux couronnes concentriques d'aubes incurvées, l'une fixe, et l'autre mobile autour d'un arbre de rotation, en agissant, à la différence de ce qui se passait dans les roues, sur la totalité, et non pas une portion de l'aubage. Volant, dans une première phase, de succès en succès, Fourneyron sut apporter la démonstration, en quelques années, des possibilités immenses et multiples qu'offrait la nouvelle machine. Celle-ci, en effet, s'avéra d'emblée capable d'atteindre un rendement aussi élevé que celui des meilleures roues existantes (70 à $80 \%$ ), de fonctionner sous de grandes variations du niveau des eaux, et de permettre un accroissement indéfini des puissances unitaires aussi bien que des hauteurs de chute : dès 1837, Fourneyron réalisa des turbines développant 220 chevaux, cependant qu'il menait à bien l'exploit, salué alors comme un prodige à travers l'Europe, d'en faire fonctionner une de 70 chevaux sous 113 et 118 mètres de chute, alors qu'il n'avait guère été possible jusque là de dépasser une vingtaine de mètres avec les roues ${ }^{6}$.

21 En dépit de ces remarquables capacités, la turbine centrifuge de Fourneyron souffrait, outre des défauts de jeunesse propres à toute innovation pionnière, d'une série de handicaps qui, à l'usage, furent de plus en plus ressentis comme préjudiciables à son utilisation industrielle. Sans même parler de son coût, qui ne la mettait guère à la portée que des plus importants manufacturiers, elle s'avérait d'une exécution difficile et d'une maintenance délicate. Son rendement, plus encore, chutait très rapidement dès que l'on s'écartait de la hauteur de chute normale pour laquelle elle avait été calculée, et elle se prêtait mal, également, aux basses chutes, d'un à quelques mètres de hauteur, qui étaient celles de la grande majorité des sites hydrauliques existants, en France notamment.

Ces limitations de la première filière en date des turbines suscitèrent, au cours des années 1830, un intense courant de recherches, qui aboutit, en France principalement, à la mise au point d'une nouvelle famille technique, celle des turbines axiales, dans lesquelles l'eau s'écoulait parallèlement à l'axe a travers deux couronnes fixes puis mobiles, non plus concentriques cette fois, mais superposées, selon une disposition proche en fin de compte du schéma originel eulérien. Deux grands types en furent développés au début des années 1840 : celui dû au mécanicien chartrain Pierre-Lucien Fontaine en 1840, et celui, breveté l'année suivante par Claude-Nicolas Jonval, contremaître chez le constructeur mulhousien André Koechlin, et qui fut véritablement rendu opérationnel par ce dernier. Les turbines de cette seconde filière ne tardèrent pas à démontrer leurs avantages pratiques sur leurs devancières de type centrifuge, du point de vue de la simplicité et de la longévité des organes mécaniques, de la facilité d'usinage, de l'abaissement des coûts de production, et de leur adaptation aux conditions topographiques dominantes en Europe, la turbine Fontaine obtenant la préférence pour les basses chutes, et celle de Jonval pour les moyennes. Si leur rendement effectif (de l'ordre de 65 à $70 \%$ ) était 
légèrement inférieur à celui des turbines Fourneyron, il se maintenait en revanche bien mieux lors de grandes variations du niveau des eaux ${ }^{7}$.

Si ces innovations mises au point en France éveillèrent les unes et les autres un intérêt aussi considérable que précoce sur tout le continent européen, force est de constater qu'elles ne se répandirent que plus tardivement dans le monde anglo-américain. Peutêtre faut-il voir là l'expression d'une conviction très assurée, de la part des techniciens et utilisateurs industriels d'Outre-Manche et d'Outre-Atlantique, dans les mérites des roues de côté de type britannique, qui leur paraissaient être parvenues, dans les deux pays, à un point de perfection difficilement dépassable.

Introduite à l'étranger - en Allemagne notamment - dès 1835, la turbine Fourneyron fit son apparition aux Etats-Unis en 1843 et celle de Jonval en 1849, après avoir été connue dès 1843-1844 en Europe continentale. Malgré leur introduction tardive, ces deux types de turbines d'origine française se répandirent massivement dans la grande industrie, en Nouvelle-Angleterre, à partir des années 1850, en sorte qu'en 1866, déjà, sur 207 moteurs hydrauliques en service dans 47 établissements cotonniers du Massachussets, on dénombrait 119 turbines inspirées du modèle de Fourneyron pour 88 roues. Dans les Etats $\mathrm{du}$ Centre-Est atlantique, ce fut la turbine Jonval qui trouva le meilleur accueil durant la même décennie ${ }^{8}$. En Grande-Bretagne, en revanche, les turbines, de l'une aussi bien que de l'autre filière, demeurèrent pratiquement inconnues jusqu'en 1850 et leur diffusion, au-delà, sans avoir été négligeable, ne semble pas avoir été comparable à celle qu'elles obtinrent globalement dans le reste de l'Europe. Il est vrai qu'à ce moment, en GrandeBretagne même - à l'exception de l'Ecosse - la page de l'hydraulique était largement en passe d'être tournée face à la marche désormais conquérante de la vapeur .

Aux Etats-Unis, cependant, le succès des turbines d'inspiration française ne dura guère plus d'une dizaine ou d'une douzaine d'années, car dès le début des années 1860 , les mécaniciens d'Outre-Atlantique développèrent une nouvelle filière originale qui s'imposa très rapidement : celle des turbines à écoulement mixte, communément évoquées ensuite jusqu'aux années 1920 sous la désignation générique de "turbines américaines », en particulier à l'étranger où elles rencontrèrent une très large diffusion.

\section{L'Amérique prend la tête : des turbines à écoulement mixte aux roues Pelton à injection tangentielle (v. 1860 - v. 1900).}

De même que les déboires rencontrés en France avec les premières générations de turbines centrifuges (joints à l'exclusivisme sourcilleux de Benoît Fourneyron dans l'exercice des droits que lui avait conférés son brevet de 1832 délivré pour une durée de 15 ans), avaient abouti à la mise au point de la filière axiale, ceux rencontrés aux EtatsUnis avec les versions pourtant plus évoluées développées sur place, y suscitèrent la réalisation d'une formule entièrement nouvelle, celle des turbines à écoulement mixte. Cette filière d'origine strictement américaine fut développée à partir d'une configuration initialement centripète apparue sous une forme très grossière en 1838 avec la roue horizontale de Howd (qui, comme telle, avec ses deux couronnes concentriques, n'en aurait pas moins été la première en date des turbines ayant vu le jour aux Etats-Unis), et dans une version nettement plus évoluée en 1848-1849 avec la machine de James B. Francis, le célèbre ingénieur en chef de la Compagnie Locks and Canals de Lowell. Mais n'ayant pas obtenu, à ce moment, des performances aussi élevées avec cette solution qu'avec les versions américanisées des filières françaises, ce dernier ne poursuivit pas 
dans cette voie, en dépit de son intérêt, illustré au début de la décennie suivante par les travaux, plus convaincants, du Professeur britannique James Thomson (frère de Sir William Thomson, l'illustre Lord Kelvin). Pour atteindre toutes ses potentialités, cette formule d'avenir se heurtait alors, pour la définition du tracé optimal des aubages, à de délicats problèmes pour l'évacuation de l'eau qui ne reçurent leur solution théorique qu'au cours du dernier quart du siècle, à la faveur des travaux de l'école mathématique germano-suisse.

Appliquant une démarche presque entièrement empirique, les mécaniciens américains, tels que Asa M. Swain, John B. Mc Cormick, James Leffel tournèrent l'obstacle en mettant au point, à partir de 1858, à force de multiples tâtonnements, une configuration composite, dans laquelle l'eau traversait la roue mobile en suivant d'abord une trajectoire effectivement centripète, puis axiale, avant d'être éjectée, le cas échéant, selon une direction centrifuge. Capable de procurer des rendements élevés - de l'ordre de $80 \%$ - et peu susceptibles de variations, de développer sans difficultés des puissances s'étageant de quelques chevaux à plus d'un millier, en acceptant d'importants débits tout en permettant de hautes vitesses de rotation, la turbine à écoulement mixte possédait, en outre, l'avantage de propriétés autorégulatrices, dues aux effets compensateurs de la force centrifuge dans une machine a éjection centrale. Elle offrait enfin l'atout d'une grande rusticité mécanique et d'une facilité d'exécution qui, jointe à la mise en oeuvre de procédés de fabrication standardisée en série relevant de l'American Manufacturing System, permit d'en abaisser radicalement le coût par rapport aux précédents types de turbines. Elle constituait, comme telle, un instrument particulièrement adapté aux besoins de la grande industrie manufacturière du Nord-Est, où elle remplaça massivement aussi bien les roues de poitrine à l'anglaise que les premières générations de turbines d'inspiration française. A partir des années 1870, les turbines à écoulement mixte, que l'on commençait déjà à désigner sur place du nom de " turbines américaines », envahirent littéralement les sites hydrauliques du Nord-Est et du Middle West, à raison de milliers et bientôt de dizaines de milliers d'exemplaires, à l'inverse de l'Europe où les turbines des filières centrifuge et axiale connurent une diffusion sans doute appréciable, mais nettement moins massive. Elles furent l'agent d'une démocratisation de la turbine, désormais accessible à toutes les catégories d'utilisateurs de l'énergie hydraulique, du plus modeste exploitant rural de moulin ou de scierie, jusqu'au grand manufacturier textile ou papetier mettant en oeuvre des milliers de chevaux ${ }^{10}$.

Si elle correspondait étroitement aux conditions de l'Est et du Centre des Etats-Unis, la turbine mixte s'avérait en revanche tout-à-fait inadaptée à celles de l'Ouest, et notamment des Montagnes Rocheuses où le boom minier suscitait, depuis le milieu du siècle, d'impérieux besoins en force motrice pour actionner, en surface ou au fond, des machineries telles que des pompes, des machines d'extraction, ou des bocards. A défaut de pouvoir employer des machines à vapeur, en raison des difficultés et du coût d'acheminement du combustible, il était indispensable, pour faire appel à l'énergie hydraulique, de disposer de moteurs tout à la fois simples et performants, et capables de fonctionner sous des chutes élevées et de faibles débits de type torrentiel. La solution majoritairement adoptée en Europe face à ce type de contraintes, sous la forme de la turbine centrifuge à admission partielle conçue en France par Louis-Dominique Girard durant les années 1850, n'était guère envisageable ici, en raison de la fragilité et du coût inhérents à cette dernière. 

point un modèle de roue à injection tangentielle, fonctionnant par impulsion, qui recevait l'eau d'une buse à haute pression. Cette machine initialement sommaire, qui avait fait l'objet d'un effort continu de perfectionnement, prit sa forme achevée au début des années 1880 à la faveur des travaux d'ingénieurs tels que Knight, W.A. Doble, et Pelton à qui la paternité en est habituellement attribuée. Ils en conçurent les aubes mobiles concaves avec leur configuration caractéristique en double coquille, et l'injecteur réglable au moyen d'un pointeau. Qualifié de roue ou de turbine selon qu'il comportait un ou plusieurs injecteurs, ce moteur n'avait rien à envier, sur le plan du rendement, aux meilleures turbines existantes ${ }^{11}$. Fille de la conquête de l'Ouest, cette innovation allait en permettre une autre, de portée universelle, celle des très hautes chutes, de plusieurs dizaines et bientôt centaines de mètres, sous des pressions supérieures à une dizaine d'atmosphères qu'avaient entreprise en Europe les recherches pionnières de Fourneyron, Girard et Berges. Ce furent les progrès de la métallurgie de l'acier qui permirent, à cette date, de disposer des matériaux capables de résister à des contraintes aussi élevées. demeurées longtemps inaperçues, ou presque, en Europe, mais plus particulièrement en France et en Grande-Bretagne. Les turbines mixtes n'apparurent vraiment de ce côté-ci de la Manche qu'à partir du début des années 1890 et le début de la décennie suivante, s'il est vrai qu'ensuite elles $y$ tinrent, pendant près d'une trentaine d'années une place dominante. En Angleterre même, elles ne rencontrèrent qu'un intérêt limité, dans la mesure où l'énergie hydraulique, au-delà du milieu du siècle, ne joua plus qu'un rôle de plus en plus marginal vis-à-vis de la vapeur, et où, d'autre part, les constructeurs britanniques, pour un marché largement orienté vers l'exportation, développèrent des turbines de conception nationale, notamment selon la formule centripète proprement dite du Professeur Thomson, qui ne paraissait guère avoir à envier à ses homologues américaines à écoulement mixte.

Largement maîtrisée et diffusée Outre-Atlantique dès les années 1880, la technologie des moteurs Pelton s'introduisit timidement au cours de la décennie suivante dans les deux pays européens envisagés ici : en Grande-Bretagne vers 1893, et en France vers 1896-1897. Outre-Manche, où les conditions topographiques en restreignaient considérablement l'emploi en dehors de l'Ecosse, ils reçurent avant 1900 une application plutôt inattendue, comme moteurs d'installations urbaines ou portuaires alimentés par des canalisations d'eau sous pression à plusieurs dizaines d'atmosphères, qui constituaient alors l'un des systèmes opérationnels encore concurrents de l'électricité pour le transport à distance et la distribution de la force motrice. En France même, les moteurs Pelton furent employés dans des stations de production électrique de montagne avant le tournant du siècle, mais ce fut seulement au cours des années 1900 que l'usage s'en acclimata véritablement. Ils commencèrent alors à être mis en oeuvre dans des conditions de fonctionnement, pour les hauteurs de chutes et les puissances atteintes, nettement plus proches de celles sous lesquelles ils étaient, depuis une vingtaine d'années bientôt, utilisés aux Etats-Unis.

Les trois cycles d'échanges techniques qui viennent d'être relatés dans leurs grandes lignes ont donc fait apparaître d'importantes disparités quant au décalage dans le temps avec lequel se sont diffusées vers les deux autres pays les différentes innovations introduites par l'un des trois, de même que de grandes inégalités quant à l'accueil que les unes et les autres trouvèrent en dehors de leur pays d'origine. Ces contrastes doivent être 
mis en rapport avec la diversité des facteurs et des agents intervenus dans le processus de transfert.

\section{Les facteurs et agents des échanges}

\section{Les facteurs indirects : la circulation de l'information technique.}

\section{L'influence de la littérature spécialisée}

Bien que les moteurs hydrauliques aient, comme de nombreux autres domaines de la technologie, donné lieu à une littérature scientifique et professionnelle de plus en plus importante à partir de la seconde moitié du XVIII ${ }^{\mathrm{e}}$ siècle, et plus encore au cours du XIV ce véhicule de l'information n'a pas du tout contribué au même degré à la transmission des différentes innovations entre les trois pays envisagés.

Les travaux de Smeaton et de ses continuateurs britanniques n'ont été que tardivement connus en France, et c'est bien davantage à l'influence des savants et techniciens français ou continentaux qu'il faut attribuer la faveur croissante dont jouirent les roues en dessus à la fin du XviII ${ }^{\mathrm{e}}$ siècle, là où il était possible de les substituer à celles en dessous. Publié pour la première fois en 1759 dans son pays d'origine, et réédité à plusieurs reprises, le traité dans lequel Smeaton dressait le bilan de ses recherches expérimentales ne fut traduit en français qu'en 1810, même si des exemplaires de l'édition anglaise de 1796 circulaient déjà en France avant cette date. L'influence des écrits de Smeaton fut, en revanche, décisive pour la propagation, Outre-Atlantique, des roues de côté, comme le reconnaissent explicitement Thomas Ellicott et Oliver Evans, auteurs du Young Millwright and Miller's Guide, publié à Philadelphie en 1795, et qui devait rester, durant des décennies, le bréviaire des constructeurs américains de moulins et moteurs hydrauliques. Si l'on s'en tient au témoignage, peu suspect, de Navier, ce serait surtout par l'entremise de ce traité américain assez tôt connu en France que les conceptions smeatoniennes se seraient diffusées, bien davantage que par le canal de la littérature d'origine britannique. Il ne semble pas que l'influence de cette dernière se soit accrue après le rétablissement de relations pacifiques et normales avec la Grande-Bretagne. L'exemple, cité par S. Chassagne, d'un Scipion Mourgues faisant venir en 1816 un grand nombre d'ouvrages anglais, en vue, notamment, de perfectionner les machines hydrauliques de son établissement, ne semble avoir guère été suivi par les autres industriels contemporains ${ }^{12}$.

La littérature technique paraît avoir joué un bien plus grand rôle, d'une manière générale, dans la propagation internationale des conceptions françaises en matière de turbines à partir des années 1830. Ce fut simplement à partir des comptes-rendus qu'il donnait des publications françaises dans le Journal of Franklin Institute et de la traduction américaine des travaux d'Arthur Morin sur la turbine Fourneyron qu'un ingénieur de Philadelphie, Elwood Morris, parvint en 1842-1843 à construire la première turbine centrifuge ayant vu le jour aux Etats-Unis.

Dans le sens inverse, s'agissant des turbines à écoulement mixte, les transferts transatlantiques n'empruntèrent guère la voie de la presse et de l'édition. Les travaux de James B. Francis avaient pourtant été assez précocement connus en France dans les milieux spécialisés, dès les années 1850, et les éditions successives de ses célèbres Lowell Hydraulic Experiments parus initialement en 1855 avaient eu une certaine diffusion. Mais les contemporains paraissent avoir été plus sensibles à la démonstration qu'il apportait 
du bien-fondé des conceptions françaises de Fourneyron et de Jonval telles qu'elles étaient reprises aux Etats-Unis, ainsi qu'à l'impressionnante maîtrise manifestée par les ingénieurs américains dans la mise en oeuvre d'aménagements hydrauliques d'une ampleur inconnue en Europe, et qu'à la présentation des formules centripètes et mixtes essayées et utilisées opérationnellement Outre-Atlantique. La plus importante tentative pour faire connaître en France ces développements technologiques du Nouveau Monde fut constituée par la série d'articles, particulièrement informés, que publia en 1883 dans la revue La Lumière Electrique, Gustave Richard, l'influent président du Comité de Mécanique de la Société d'Encouragement pour l'Industrie Nationale. Pourtant, en dépit de l'autorité incontestée de son auteur, qui soulignait les nombreux avantages des solutions mises au point en Amérique, cette publication ne paraît guère avoir incité, à court terme, les constructeurs et industriels utilisateurs français à les reprendre à leur compte. Lors du dernier épisode, celui de l'introduction du système Pelton, l'impact des périodiques professionnels, qui, au début des années 1890, prirent soudainement conscience des possibilités ouvertes par cette formule à l'heure où la conquête des très hautes chutes venait enfin à l'ordre du jour en France, ne peut être évalué isolément, dans la mesure où, au même moment, d'autres canaux la firent connaître plus tangiblement, à commencer par les grandes Expositions.

\section{Limpact des grandes Expositions}

Forme de confrontation technique et commerciale spécifiquement issue de la révolution industrielle, les grandes expositions n'ont pris une place majeure dans les échanges technologiques internationaux qu'à partir du milieu du siècle, avec l'avènement des expositions dites universelles, auxquelles toutes les nations étaient invitées à participer, à la différence de la période antérieure où, comme ce fut le cas en France, s'étaient succédé selon une périodicité régulière des manifestations restreintes aux seuls producteurs nationaux. Alors que ces dernières s'étaient tenues dans un contexte étroitement protectionniste, les expositions universelles participaient des efforts pour promouvoir le libre-échange, et partant, encourageaient explicitement la circulation internationale des technologies.

En dépit de ces intentions, les effets des expositions postérieures à 1850 dans le domaine envisagé ici se sont avérés très inégaux. La première exposition londonienne de 1851, dans laquelle les moteurs hydrauliques tinrent, au pays d'origine de la machine à vapeur, une place des plus restreintes, fut l'occasion pour les techniciens et constructeurs d'Outre-Manche d'une véritable révélation des potentialités offertes par les turbines, à la lumière des réalisations françaises en la matière. Aussi bien, la turbine Fontaine construite par la Maison Fromont et Fils de Chartres, l'un des très rares spécimens de la technologie hydraulique moderne présentés à cette occasion, se vit-elle décerner la médaille du Conseil, l'une des plus hautes distinctions attribuées à Crystal Palace. Ce fut seulement à la suite de cette exposition que plusieurs constructeurs britanniques (BryanDonkin, Williamson Brothers devenue par la suite Gilbert Gilkes and Gordon, North Moor Foundry) entreprirent de fabriquer à leur tour des turbines, soit en s'inspirant des formules éprouvées venues du continent, soit même en développant des solutions originales, comme celle de la turbine Thomson évoquée plus haut. La présentation, en revanche, de cette innovation britannique, lors de la seconde exposition universelle parisienne en 1867, ne semble pas avoir convaincu les techniciens français de l'intérêt de la filière centripète, en dépit de l'estime que lui témoigna le rapporteur du jury. 
39 Si peu de techniciens français prêtèrent une suffisante attention aux moteurs hydrauliques de facture américaine, pourtant nombreux, présentés en 1876 lors de l'exposition du Centenaire des Etats-Unis à Philadelphie, il n'en alla pas de même de l'Exposition Universelle de Chicago en 1893. En ce domaine comme dans tant d'autres, celle-ci fut l'occasion pour les industriels français de ressentir, pour la première fois, dans toute son ampleur, l'avance technologique prise par la République américaine et tout autant le fossé qui se creusait sur ce plan avec certains pays du Vieux-Continent, à commencer par la France. Cette prise de conscience, parfois brutale, transparaît clairement dans l'important rapport que Gustave Richard rédigea sur le sujet, à la demande du gouvernement français. Elle contribua de façon décisive à accélérer l'adoption, par un grand nombre de constructeurs français, de la turbine mixte que, jusque là, seuls les Frères Singrun d'Epinal avaient commencé à produire depuis 1890 . Ce fut ainsi à la suite de sa visite à Chicago, en 1893, dans le cadre du voyage de la Société Française des Ingénieurs Civils, que Francis Brault, l'un des dirigeants de la firme chartraine considérée comme le numéro un dans la branche, s'empressa de déposer un brevet adaptant aux turbines mixtes un certain nombre de dispositifs propres à l'entreprise, de manière à en naturaliser la fabrication. Plus encore, cette exposition révéla véritablement la turbine Pelton aux techniciens français, en sorte que la construction ne tarda pas à en être engagée aussi de ce côté-ci de l'Atlantique.

\section{Le rôle prépondérant des formes directes de transfert technique}

\section{Les exportations de technologie}

40 A l'ère de l'industrialisation, la transmission des techniques s'est exercée, dans une proportion croissante, par le canal des ventes directes. Ces exportations de technologie ont rapidement tendu à prendre leur double forme moderne, celle de la vente d'équipements et celle de la cession de brevets.

41 L'exportation de moteurs hydrauliques, inconnue à l'époque antérieure où ceux-ci, principalement exécutés en bois, étaient réalisés sur les lieux mêmes de leur installation, fut la conséquence de l'industrialisation de leur fabrication liée à l'avènement de la construction métallique. Elle concerna par conséquent les turbines, mais aussi les roues, particulièrement lorsque celles-ci atteignaient de grandes dimensions. S. Chassagne signale ainsi l'importation en France, après 1815, par des manufacturiers du secteur cotonnier, d'un certain nombre de roues à augets métalliques de facture typiquement britannique: ainsi, parmi les manufacturiers les plus importants de Haute-Alsace, les Haussmann en acquirent-ils une en 1825 pour leur établissement du Logelbach, ou encore, l'année suivante, la Maison N. Schlumberger et Cie alla-t-elle se procurer OutreManche la grande roue destinée à ses usines de Wesserling, sur laquelle les ingénieurs militaires procédèrent à une série d'expériences appelées à un grand retentissement.

En sens inverse, les exportations directes de turbines françaises vers les deux autres pays envisagés ici semblent avoir été beaucoup plus rares. Les achats de quelques turbines Jonval-Koechlin et Fontaine effectués en 1849 et 1851, auprès de leurs constructeurs français respectifs, par la maison britannique Bryan-Donkin semblent, rétrospectivement, avoir eu pour but essentiel de lui permettre de se familiariser avec la technologie de ce type de moteurs hydrauliques, qu'elle connaissait encore mal, avant de se lancer à son tour dans ce genre de fabrications, selon une démarche devenue classique dans le 
processus de naturalisation par un pays de techniques importées. Les ventes de ces mêmes machines aux Etats-Unis furent, quant à elles encore plus rares, dans la mesure, en particulier, où la firme mulhousienne eut recours à une autre formule pour s'implanter Outre-Atlantique. Les exportations directes des Etats-Unis vers la France et la Grande-Bretagne ne paraissent pas, non plus, avoir tenu une place capitale pour la diffusion, durant les années 1880 , des turbines à écoulement mixte. A défaut d'avoir compté quantitativement, elles eurent cependant une indéniable valeur d'exemple pour le reste de l'industrie. Ainsi la firme John Turnbull de Glasgow servit-elle de revendeur, pour la Grande-Bretagne, de la turbine «Hercule » inventée par John B. Mc Cormick et construite par la Holyoke Cy (elle équipa l'importante papeterie d'Aberdeen qui disposait ainsi de 1200 chevaux), cependant qu'en France la maison Sloan et Cie assura, à partir de 1885, l'importation exclusive des turbines produites par plusieurs constructeurs américains.

En matière de transferts transatlantiques, ce sont en fait les exportations invisibles qui ont eu la plus grande importance, avec le développement de la pratique des cessions de licences, rendues possibles dans le cas français par la réforme de la législation sur la propriété industrielle introduite en 1844. Le recours à cette formule fut favorisé par le facteur de la distance, qui allongeait considérablement les délais de livraison, de même, bien entendu, que les prix des machines rendues sur le lieu d'utilisation. Avec des équipements tels que les moteurs hydrauliques, la proximité entre le fabricant et la clientèle constitue un atout majeur dans l'obtention des commandes, tant au stade de leur exécution qu'à celui du service après-vente. Ce furent apparemment des motivations de cet ordre qui incitèrent la maison André Koechlin et Cie à envoyer en 1849 l'un de ses associés, Emile Geyelin, ouvrir une succursale à Philadelphie, avec pour mission de concéder la licence de la fabrication et de la vente des turbines Jonval aux Etats-Unis. Celui-ci ne tarda pas à conclure de tels accords avec plusieurs des principales entreprises de constructions mécaniques de la côte Est, telles que I.P. Morris à Philadelphie, ou la West Point Foundry près de New-York. Il assura une large publicité à la turbine Jonval en en faisant installer un exemplaire à la station élévatoire de la ville de Philadelphie, qui démontra l'aptitude, indispensable pour la continuité du service public, de ce type de moteur à fonctionner aussi bien en période de crues que de basses eaux. Le prestige de la turbine d'origine mulhousienne fut à son comble lorsqu'au détriment de concurrents américains, Emile Geyelin remporta en 1859-1860 le concours organisé pour l'équipement des Fairmount Waterworks. Dans l'autre sens, des cessions de licences intervinrent également pour l'exploitation, en France, des brevets que des firmes ou des inventeurs américains y déposèrent, en nombre constamment croissant au cours des années 1880 et 1890 , à propos de turbines mixtes ou de roues tangentielles à haute pression du genre Pelton. Ce fut ainsi que l'entreprise Singrun Frères d'Epinal acquit la licence du brevet relatif à l'un des modèles les plus performants de turbine mixte, mis au point aux EtatsUnis sous la marque « Hercule » par John B. Mc Cormick.

\section{Les voyages et missions d'information à l'étranger}

Le rôle, pour l'introduction en France des principales innovations britanniques apparues au XVIII ${ }^{e}$ siècle, des missions de renseignements Outre-Manche, relevant souvent de l'espionnage industriel, qui furent exécutées à la fin de l'Ancien Régime, à titre privé ou pour le compte du gouvernement, par des experts ou des industriels, a été depuis longtemps mis en lumière essentiellement par l'oeuvre pionnière de Charles Ballot, 
complétés depuis par Bertrand Gille dans le cas de la métallurgie ou Serge Chassagne dans celui de l'industrie cotonnière. Dans tous les cas, cependant, l'intérêt s'était à l'époque porté sur les équipements de production de branches déterminées et les machines à vapeur; il semble que l'on ait alors négligé les progrès que les techniciens britanniques étaient également en train d'apporter à la technologie des moteurs hydrauliques, dans la mesure où ceux-ci n'étaient pas aussi spectaculaires, extérieurement, que ceux intervenus dans les autres domaines, et où ce secteur semblait relever d'un savoir-faire bien établi et depuis longtemps banalisé. Il n'en alla plus tout-à-fait de même au début du siècle suivant, lorsque l'on commença à se rendre compte que, sur ce plan aussi, il y avait beaucoup à apprendre de l'Angleterre, au moment précisément où les roues de côté $\mathrm{y}$ atteignaient leur maturité.

Après 1815 , les industriels et techniciens français qui purent reprendre le chemin de la Grande-Bretagne pour étudier sur place la mise en oeuvre dans les usines des nouveaux procédés de fabrication, commencèrent à inscrire à leur programme le perfectionnement de ce que l'on appelait alors "les hydrauliques ". Le même Scipion Mourgues que nous avons vu plus haut faire venir en 1816 nombre d'ouvrages techniques britanniques, y compris dans le domaine hydraulique, déclare avoir envoyé ensuite des informateurs en Angleterre vérifier le bien-fondé des assertions figurant dans la littérature. Au cours de la période suivante, le caractère excitant de l'innovation majeure constituée par les premières générations de turbines attira en France nombre de spécialistes étrangers. $\mathrm{Ce}$ fut ainsi à la suite d'un voyage en France de l'industriel cotonnier Andrew Robeson Jr, au cours duquel il avait vu fonctionner une turbine Fourneyron dans un établissement d'indiennage, sans doute alsacien, que la firme de constructions mécaniques E.C. Kilburn, établie à Fall River (Massachussets), entreprit, à partir de 1844, de fabriquer à son tour des turbines de ce type. Durant ces mêmes années, l'ingénieur civil britannique William Cullen vint en France se renseigner sur les turbines centrifuges, mais s'étant heurté au refus de Fourneyron de lui communiquer toute espèce d'information, il en fut réduit, de retour en Irlande, à s'appuyer sur la littérature technique pour concevoir des machines de cette configuration.

\section{Les migrations de techniciens}

Durant la première phase du processus, ce furent surtout les migrations internationales de techniciens - forme la plus directe de transfert de savoir faire - qui contribuèrent le plus activement à la propagation des roues perfectionnées d'origine britannique.

La venue aux Etats-Unis de spécialistes anglais représente un fait tellement général de l'histoire américaine que l'historiographie d'Outre-Atlantique ne semble pas avoir éprouvé jusqu'ici le besoin d'identifier, au milieu de la masse de travailleurs qualifiés ayant traversé l'Océan au début du siècle dernier, ceux qui y apportèrent la nouvelle technologie des moteurs hydrauliques dérivée en droite ligne des travaux de Smeaton et de ses continuateurs. Il n'en va plus de même aujourd'hui dans le cas français, où l'essor des études sectorielles, de caractère régional ou national, motive un effort accru pour mieux repérer ceux qui furent concrètement les introducteurs sur le terrain de ce que les contemporains désignaient du terme générique de « techniques anglaises $»^{13}$.

Il paraît maintenant établi qu'en matière d'hydraulique, les premiers éléments de ces techniques pénétrèrent véritablement en France, au début des années 1800 à la faveur du court répit qui suivit la Paix d'Amiens. Le rôle historique de premier initiateur en date 
revient ici à William Aitken, appelé sur le continent en Frimaire An x à la demande de l'industriel d'origine britannique Henry Sykes, qui avait créé en 1792 une filature mécanique de coton à Saint-Rémy-sur-Avre (Eure-et-Loir), et auquel devait succéder son gendre William Waddington, fondateur de la célèbre dynastie de manufacturiers et d'hommes politiques français ${ }^{14}$. Sous l'Empire, Aitken ouvrit un atelier de constructions mécaniques à Senonches, dans le même département, où il pouvait disposer, sur place, d'un approvisionnement en fontes du Pays d'Ouche se rapprochant, par leurs qualités, de celles utilisées en Grande-Bretagne pour l'obtention par moulage de pièces de mécanique. Naturalisé français en 1810, il équipa au même moment, suivant les principes anglais, les installations hydrauliques de plusieurs des plus importants établissements textiles français de l'époque napoléonienne, à commencer par ceux d'Oberkampf à Jouy-en-Josas, Richard-Lenoir, ou encore Germain-Ribouleau à Louviers.

Après 1815, les techniciens britanniques affluèrent en France, où un certain nombre d'entre eux commencèrent à construire des roues hydrauliques conformes aux principes prévalant désormais Outre-Manche. On peut citer ainsi, pour la fourniture de l'industrie textile locale, le cas d'un James Barker, embauché d'abord au service des Sykes et des Waddington, puis établi à son compte à Petit-Quevilly près de Rouen, où il s'associa par la suite à ses deux compatriotes William Sudds et Benjamin Adkins; ou encore, dans le cas du Haut-Rhin, celui d'un Job Dixon, créateur en 1817 aux côtés des alsaciens Risler, d'un atelier ayant livré quelque 43 roues aux manufactures de la contrée de 1820 à $1828^{15}$. Il en alla de même pour l'industrie sidérurgique, avec le grand établissement édifié en 1824 à Charenton-le-Pont, aux portes de Paris, par Manby et Wilson, qui réalisa ainsi en 1823 la grande roue motrice de la forge à l'anglaise de Sainte-Colombe-sur-Seine. Ce fut auprès de tels mécaniciens d'origine britannique installés comme constructeurs sur cette rive-ci de la Manche que se formèrent nombre de techniciens français ayant ensuite fondé leurs propres entreprises. Ces derniers y apprirent la technologie des roues hydrauliques à l'anglaise en même temps que celle d'autres équipements industriels, machines à vapeur et appareils de fabrication destinés à des branches déterminées. La venue sur le continent des spécialistes anglais fut sans aucun doute le facteur le plus décisif pour l'acclimatation du nouveau savoir-faire en matière de roues hydrauliques, parallèlement à celle des innovations métallurgiques qui, sur un plan général, conditionnèrent la mise au point des équipements énergétiques de l'âge industriel.

Les relations techniques entre les trois pays qui ont donné successivement le ton à l'évolution internationale des moteurs hydrauliques constituent un cas, parmi d'autres, qui incite à revoir la vision, naguère encore illustrée par la synthèse d'un D.S. Landes, qui tendait à ramener la première industrialisation à une extension progressive aux autres pays aujourd'hui développés du corpus des innovations techniques et du modèle de croissance britanniques. Il montre qu'en l'espèce, il n'y eut pas de transfert unilatéral d'un pays initiateur à des pays suiveurs, mais plusieurs pôles nationaux d'innovation, qui ont joué chacun à leur tour un rôle tout aussi actif et décisif vis-à-vis du progrès général du secteur concerné, tout en entretenant entre eux (et avec d'autres) des échanges continus, en dehors des phases de conflit armé de l'ère révolutionnaire et impériale, où ils furent en grande partie interrompus.

51 A chaque fois, l'initiative d'une filière technique, dans le domaine étudié ici, fut suscitée dans un pays donné par une demande économique interne étroitement liée aux structures et à la nature des activités d'entraînement de l'appareil productif, et orientée par des conditions spécifiques tenant largement, dans le cas présent, à des contraintes 
géographiques, d'ordre hydrologique notamment. Avec un temps de latence très variable, la portée internationale, sinon universelle, de chacune de ces innovations ayant été reconnue, pour un temps, elles furent chacune à leur tour reprises dans un certain nombre d'autres pays. Il n'en demeure pas moins que certains pays suiveurs firent plus que simplement acclimater chez eux les techniques - en l'occurrence les types de moteurs - venues du pays initiateur; ils les incorporèrent à leur système technique national et les naturalisèrent en les adaptant à leurs conditions propres et en leur ajoutant, le cas échéant, des perfectionnements originaux : ce fut ainsi le cas avec la facture semi-métallique, en France, des roues de côté, avec la version américanisée de la turbine Fourneyron conçue par un Uriah A. Boyden, ou encore la francisation de la turbine mixte opérée par la firme de Chartres qui lui appliqua la formule maison du pivot supérieur.

Aussi bien, donc, en amont qu'en aval du processus d'innovation, à son origine que dans son extension, s'affirment ici encore tout à la fois le caractère fondamentalement international du mouvement de l'innovation technique et la diversité des voies nationales. Variété des solutions promues par chacun des pays initiateurs, mais variété aussi des formes sous lesquelles les pays suiveurs les ont reprises. Ceux-ci tendirent, en fait, à n'acclimater que certaines composantes de la technologie du pays leader : ainsi, la France ignora-t-elle, à de rares exceptions près, la formule des roues à réaction, dites de Barker et de Parker, qui connut pourtant un réel succès et une importante carrière opérationnelle en Grande-Bretagne et plus encore aux Etats-Unis (et aussi, dans une moindre mesure, en Europe centrale). De même, les développements technologiques importants apportés en France aux roues verticales fonctionnant sous de basses chutes, depuis celle de Poncelet, apparue en 1824-1825, jusqu'à celle de Sagebien mise au point en 1849-1850, qui trouvèrent un large écho dans une partie du continent européen, n'eurentils que très peu d'influence dans le monde anglo-américain, tout comme la formule, pour l'exploitation des très hautes chutes, de la turbine Girard à admission partielle au contraire largement employée avant 1900 en Suisse, en Allemagne et en Italie. Ce furent, d'autre part, le plus souvent les limitations rencontrées dans la mise en oeuvre opérationnelle, dans un pays déterminé, des solutions, à l'origine plus avancées, venues de l'étranger, qui conduisirent à en développer de nouvelles: ainsi certaines inadaptations aux conditions de l'Hexagone des roues perfectionnées à l'anglaise stimulèrent-elles en France l'apparition des turbines centrifuges et axiales, comme celle des turbines mixtes fut favorisée aux Etats-Unis par les insuffisances auxquelles donna lieu l'exploitation de ces dernières.

Au-delà de la période envisagée ici, le processus international d'innovation a, toutefois, trouvé son terme en matière de moteurs hydrauliques, avec l'affirmation, entre 1880 et 1920, d'un quatrième foyer initiateur, situé cette fois en Europe centrale, dans l'aire de culture germanique, englobant la Suisse, l'Allemagne et les pays tchèques et autrichiens. Il fut à l'origine, d'une part, de la mise au point définitive, entre 1880 et 1900 , de la turbine centripète pure, connue de par le monde après 1920 sous le nom de turbine Francis, en hommage aux anticipations du grand ingénieur de Lowell, et, d'autre part, de la création vers 1915 d'une formule originale, la turbine-hélice, due à Viktor Kaplan, qui apportait la solution définitive au problème de l'exploitation des grands potentiels fluviaux. Les développements techniques de ces quelque quarante années aboutirent de la sorte à la sélection durable des trois grands types de moteurs hydrauliques dont l'usage est devenu véritablement universel au $\mathrm{Xx}^{\mathrm{e}}$ siècle jusqu'à aujourd'hui : la roue-turbine 
Pelton pour les très hautes chutes, la turbine Francis pour les moyennes, et la turbine Kaplan pour les plus basses.

Même si la France a repris aujourd'hui sa place parmi les grands constructeurs mondiaux de moteurs hydrauliques, ses apports historiques et ceux de la Grande-Bretagne, qui avaient été si importants durant la majeure partie du XIXe siècle, semblent avoir été effacés dans ce domaine de la technologie, alors que ceux des Etats-Unis subsistent très largement, participant de la suprématie technique générale qu'ils ont acquise depuis la fin du siècle dernier.

A partir de ce cas d'espèce, il convient, pour conclure, de s'interroger sur la pertinence, appliquée à la technologie, de la notion d'aire culturelle au sein même de la civilisation occidentale initiatrice de l'industrialisation. Ainsi l'influence du facteur linguistique pourrait-elle rendre compte, au moins en partie, de la dissymétrie des échanges entre la France et le monde anglo-américain. La communauté de langue, liée à un même fonds de population, a certainement contribué à former, en ce domaine comme dans d'autres, la similitude des solutions développés en matière de roues hydrauliques, jusqu'au milieu du siècle dernier tout au moins, par les techniciens britanniques et américains. L'obstacle linguistique, à l'opposé, n'est probablement pas étranger au retard d'ensemble avec lequel les innovations apportées successivement par ces derniers s'acclimatèrent en France, tout comme, en sens inverse, le rayonnement persistant de la langue française a facilité la connaissance, aux Etats-Unis notamment, des avancées scientifiques et techniques intervenues de ce côté-ci de la Manche entre 1820 et 1850. Il reste que ce sont tout autant des aires de culture technique qui se dessinent à travers cet exemple, témoignant, au-delà de la langue, et parfois malgré celle-ci, de mentalités similaires concernant les rapports entre théorie et pratique, et unifiant ces dernières, de voies analogues dans les filières de formation intellectuelle et professionnelle des techniciens. Ces faits de culture ne sauraient, de toute manière, faire perdre de vue, s'agissant d'échanges techniques, l'intervention souvent déterminante de mécanismes d'ordre économique et financier, en raison de l'importance, dans le cas de la Grande-Bretagne et de la France au siècle dernier, des exportations de capitaux investis directement dans l'industrie auxquelles ces transferts furent souvent associés, suivant les rythmes et l'amplitude dégagés par les analyses classiques de Leland H.Jenks et de Rondo E. Cameron.

\section{NOTES}

1. L'Acquisition des techniques par les pays non-initiateurs. Pont-à-Mousson, 28 juin/5 juillet 1970, Colloque international du C.N.R.S. organisé conjointement avec l'I.C.O.H.T.E.C., Paris, Éditions du C.N.R.S., 1973, $624 \mathrm{p}$.

2. Louis C. HUNTER, A History of Industrial Power in the United States, 1750-1930, Vol. I: Waterpower in the Century of Steam, Charlottesville, The University Press of Virginia for the Eleutherian MillsHagley Foundation, 1979, 606 p. 
3. John SHAW, Water Power in Scotland, 1550-1870, Edinburgh, John Donald Publishers. 1984, $\mathrm{XI}-606 \mathrm{p}$.

4. Terry S. REYNOLDS, Stronger than a Hundred Men. A History of the Vertical Water Wheel. Baltimore and London. The John Hopkins University Press, 1983, XVIII-454 p. Cf. sur ce point, le ch.4, pp. 218-233.

5. Ibid., ch. 5, pp. 266-320.

6. ATELIERS CROZET-FOURNEYRON, Centenaire des Ateliers Crozet-Fourneyron, 1832-1932, Paris Poyet-Fourneyron, 1932.

7. Sur les turbines axiales françaises du second tiers du XIX siècle, voir l'étude de Serge BENOIT, Geneviève DUFRESNE et Gérard EMPTOZ (avec la collaboration de Jean-Paul GUIARD), «Une production de pointe dans une entreprise innovante: les turbines Fontaine au temps des fondateurs de la Maison de Chartres. 1837-1873 ", à paraître in Cahiers de la Société française d'Histoire des Sciences et des Techniques.

8. L.C. HUNTER, op. cit. note 2, ch. 7, « The Hydraulic Turbine », pp. 292-342.

9. G.N. von TUNZELMANN, Steam Power and British Industrialization to 1860, Oxford, Clarendon Press, 1978, XII-344 p.

10. L.C. HUNTER, op. cit. notes 2 et 8 , ch. 8 , «A Generation of Declining Use and Advancing Technology », pp. 343-415.

11. Camille KRANTZ éd., Exposition internationale de Chicago en 1893. Rapports. Comité 15. La mécanique générale américaine à l'exception de Chicago, Premier volume, Paris, Imprimerie Nationale, 1894, pp. 201-244. Exposition universelle internationale de 1900 à Paris. Rapports du Jury international. Groupe IV. Matériels et procédés généraux de la mécanique. Deuxième partie, Classe 20, Paris, Imprimerie nationale, 1904, pp. 144-256.

12. Serge CHASSAGNE, La naissance de l'industrie cotonnière en France: 1760-1840. Trois générations d'entrepreneurs, Thèse de Doctorat sous la direction de Louis BERGERON. Paris, E.H.E.S.S., 1986, 3 vol. dactylogr.

13. Cf. l'article de Serge BENOIT, Gérard EMPTOZ et Claudine FONTANON, " Une banque de données en développement sur la mécanisation de la France », paru dans le numéro 3 des Cahiers du C.R.H., pp. 15-26.

14. Communication inédite, aimablement communiquée par l'auteur, de Geneviève DUFRESNE à la Ve Conférence internationale sur la Conservation du Patrimoine industriel, Boston-Lowell, 1984, intitulée «Technology Transfers from England to France. The Waddington's Cotton Factories, as an Example in the Avre Valley (1792-1850)».

15. S. CHASSAGNE, op. cit. note 12 , passim.

\section{AUTEUR}

\section{SERGE BENOIT}

Serge Benoit est chargé de recherche au Centre de Recherches Historiques. 